\title{
Can positron emission tomography work in the African tuberculosis epidemic?
}

\author{
Mike Sathekge and John Buscombe \\ Nuclear Medicine, University of Pretoria, Republic of South Africa
}

\begin{abstract}
Summary
The greatest health problem in sub-Saharan Africa is the complex of HIV and TB. PET has been primarily developed to look at cancer but can PET be used for this major health problem. F-18FDG has a high sensitivity in TB but the pattern of uptake is not specific. Attempts to improve the specificity of F-18 FDG imaging in the context of TB by using dual time scanning has not proved useful. There is evidence that F18 FDG can be used to monitor the effectiveness of anti-tubercular therapy but the number of patients studied remains small. What is needed is the development of affordable tracers which are more Sensitive and specific to TB maybe using generator produced products such as Ga-68
\end{abstract}

\section{Introduction}

Much is written about transfer of technology from developed to developing countries. In the area of health care not only is there the issue of poverty within the developing countries but the pattern and epidemiology of disease can be very different. South Africa is in a unique position in that it has a well off middle class with access to some of the best health care that can be bought but the majority of the population tend suffer from the diseases of poverty and access to health care may depend on a number of factors including the educational level and expectations of the patients themselves.

South Africa suffered the epidemic of HIV/AIDS later than Eastern Africa but now has some of the highest rates of HIV infection in the world with no clear evidence of the present epidemic abating. In addition there has re-emerged one of the diseases that was originally thought to be in decline which is TB. Coexistent infection often with multi-drug resistant TB and HIV has become for many the defining illness in Southern Africa in the early $21^{\text {st }}$ century. Therefore is there a place for an expensive high technology tool such as PET imaging in this scenario. Is PET essential for these patients or is it seen as an extravagance which should be reserved only for the rich.

\section{The problem}

It is reported that South Africans suffer the highest incidence of HIV ( $35 \%$ of the population) and the second highest incidence (at least $4 \%$ of the population) of TB world wide $(1,2)$. Access to healthcare is uneven with pockets of extreme poverty and poor housing in both rural and urban setting especially with crowded informal housing $(3,4)$. In addition there is increasing incidence of drug resistant TB requiring more complex dosing and prolonged dosing regimes (5). Therefore there is an increasing need for effective imaging not only to diagnose TB within sub-Saharan Africa but within follow-up of these patients. This may have to be done with the financial constraints of a developing country. Superficially it would appear that a technology such as PET would not be suitable for such a clinical problem. However where such machines exist it would be perverse if this technology was not applied to the greatest public health issue of the early $21^{\text {st }}$ century in Southern Africa

\section{2-Fluorodeoxy-D-glucose imaging}

Infective sites and activated leucocytes of both granulocytic and lymphocytic origin have increased utilization of glucose and it has been known for over 10 years (6-9) In one of these papers it was noted that TB could have a high uptake of FDG with SUVs in the range of 1.9-3.7 where there is significant overlap with bronchogenic carcinoma. Focal F-18 FDG uptake in a tuberculous nodule has been reported as a cause for false positive F-18 FDG PET being used to look for malignant pathology $(10,11)$. One report from the PRC showing how the images performed in patients with widespread TB can be mistaken for disseminated malignancy. 
However if F-18 FDG is being used to identify the presence of TB then the test can be useful with a sensitivity for TB recorded as high as $90 \%$ (8). This level of accuracy will depend on reading the scan with the knowledge that TB is expected. The level of suspicion will depend on the incidence and presentation of

the disease. A different index of suspicion will exist in suburban United States than Urban South Africa and successful reporting of the study will require a good local knowledge on how the disease presents and in prevalence. For example in a South Asian community TB has a high prevalence but tends to present as respiratory disease (11). At present in sub-Saharan Africa TB tends to co-exist with HIV/AIDS and it may have a variety of presentations including nodal disease where there maybe a differential diagnosis of lymphoma.

\section{Patterns of uptake}

In the UK and many other developed nations F-18 FDG imaging is performed as part of the assessment of patients with radiological abnormalities such as a single pulmonary nodule. The pattern of uptake seen tends to involve an area of intra-thoracic lesion with and without associated lymphadenopathy (11). In the South African situation there are different considerations though TB is common in the HIV/AIDS population it is not an exclusive relationship and many patients who are HIV negative are at risk of TB partly due to poor living conditions and crowded urban areas. In addition HIV itself leads to patterns of F18 FDG uptake that may mimic infection including cervical and thoracic lymph node uptake during the initial infection. Through this tends to be symmetrical and follows a step wide progression form neck to groin (12). A similar pattern may be seen with TB in HIV positive patients but the pattern of uptake may be less symmetrical and there may also be lung parenchyma involvement. The overall appearance is that of multiple sites of involvement with intense uptake of F-18 FDG scattered throughout the body from neck to pelvis. In addition in HIV patients there may be sub-cutaneous uptake of F-18 FDG in sites of lipodystrophy itself related to anti-retrorviral treatment (13). Therefore in a situation where a patient is imaged where they may have TB and new HIV infection it may not be possible to determine the exact cause of F-18 FDG uptake at any particular site. The situation can be further complicated by the late presentations of some patients and the presence of associated malignant disease such as lymphoma which itself in sub-Saharan Africa can be related by co-infection such as EBV(14).

\section{Dual-phase 2-fluorodeoxy-D-glucose imaging}

In some patients there may be a question as whether pulmonary uptake is malignant or benign in particular is it possible to differentiate between $\mathrm{Tb}$ and tumour. The general basis of this theory is that though both may have an initially high SUVmax malignant tissues would have a greater retention, between 1 and 2 hours of FDG than benign processes such as TB. This was tested in a prospective series of 31 suspicious pulmonary lesions $60 \%$ of the benign lesions including 10 due to TB had a retention index greater than $10 \%$ compared to $62 \%$ in malignant disease, the inference being that late imaging is not useful. (15). This work has been conformed in a Sub-Saharan population in a group of 30 patients with a high incidence of TB the SUVmax in the benign group was higher than those with cancer (16), which meant that is a traditional SUVmax cut-off of 2,5 was used the specificity of F-18 FDG was only $25 \%$ for finding cancer. Again the washout of F-18 FDG as measured by change in SUVmax was almost identical in the two groups especially when TB was compared with cancer. It would appear therefore that the use of washout rates for F-18 FDG is unlikely to helpful in differentiating TB from other causes of FDG uptake in the sub-Saharan patient.

\section{2-Fluorodeoxy-D-glucose and treatment monitoring}

The treatment of TB can be problematic, the disease itself may be resistant to standard treatment and treatment itself is prolonged with regimes in HIV positive patients being anywhere from 3-9 months. Hospitalization for this period of time is not possible so treatment is performed as an out-patient. However this raises the problem of compliance especially if the patient feels better. The timing for a course of treatment is empirical and tends to be the maximum needed to treat $\mathrm{Tb}$ in that clinical situation. However there can be great variation in the timing of the required treatment so an objective method to monitor treatment is required. Using an animal model and TB infection model it has been shown that quantitative F-18 FDG uptake can be used to monitor the effectiveness of treatment (17). In this study there was a clear 
correlation between fall in F-18 FDG uptake in the lungs and successful administration of a bacteriocidal drug. In contrast uptake of F-18 FDG increased when an ineffective drug was given and in the control group.

However clinical data remains sparse a recent study showed that the SUVmax in tuberculose multiple lymph nodes returned to normal in a small series of 3 patients but he time scale again emphasized the variability of treatment in that it took between 182 and 315 days (18).

\section{Other tracers}

There is little published work looking at new tracers for PET. This is primarily due to the slow development of non F-18 FDG techniques. Whilst Ga-67 citrate has been used to find infection including TB in HIV positive patients $(19,20)$ The short half life precludes the use of this agent as it takes 24 hours to fully bind in vivo to the transit proteins such lactoferrin and transferrin which are involved in the targeting of infection. This is clearly not practical with the 68 minute half life of Ga-68. It has been suggested that Ga-68 could be complexed to one of these proteins but as yet the only work had been with Ga-68 transferrin to look at the integrity of the alveolar membrane (21). An alternate approach would be to use labeled leucocytes using F-18 FDG for cell labeling however there may be two significant problems, firstly the safety of staff handling HIV positive blood and secondly the integrity and functioning of labeled leukocytes in HIV positive patients as it has been found that labeled leucocyte studies may not be useful (22).

A different approach would be the use of radiolabelled antibiotics the most obvious candidate would be isoniazid and a single photon Tc-99m labeled has been used in rats but only for imaging tumours (23). However such as approach maybe labeled with F-18 or Ga-68 could produce a more TB specific agent though the level of uptake compared with uptake in tumour could be problematic.

A further alternative which offers the possibility of rapid infection specific imaging is based on the natural anti-microbial peptide ubiquitin. Ubiquitin itself attaches to mycobacterium and appears to be involved in the process of autophagy resulting in internalization of the microbe within inflammatory cells (24). Therefore as this is a specific and fairly rapid process may be a way to identify the presence of myocabterium in vivo especially if labeled with a radiometal such as $\mathrm{Ga}-68$ or $\mathrm{Cu}-64$ which unlike F-18 will not be effluxed from the cell after phagocytosis. These methods would have the advantage of an easily prepared radiopharmaceutical using a generator produced positron emitting radionuclide and rapid imaging within 60 minutes of injection.

\section{Conclusion}

There is an increasing body of evidence that PET can be useful for both diagnosis and monitoring therapy response. At present the diagnosis of TB depends on the pattern of uptake seen and will do so until more specific tracers are developed.

\section{References}

1) Report on the global AIDS epidemic Geneva; UNAIDS 2008

2) Global Tuberculosis Control Report surveillance planning financing Geneva WHO 2009

3) Welz T, Hosegood V, Jaffar S, Bätzing-Feigenbaum J, Herbst K, Newell ML. Continued very high prevalence of HIV infection in rural KwaZulu-Natal, South Africa: a population-based longitudinal study. AIDS. 2007;21:1467-72

4) Wood R, Liang H, Wu H, Middelkoop K, Oni T, Rangaka MX, Wilkinson RJ, Bekker LG, Lawn SD Changing prevalence of tuberculosis infection with increasing age in high-burden townships in South Africa Int J Tuberc Lung Dis. 2010;14:406-12

5) Dheda K, Warren RM, Zumla A, Grobusch MP. Extensively drug-resistant tuberculosis: epidemiology and management challenges Infect Dis Clin North Am. 2010;24:705-25

6) Bakheet SM, Powe J, Ezzat A, Rostom A. F-18-FDG uptake in tuberculosis Clin Nucl Med. 1998;23:739-42 
7) Jeong YJ, Lee KS. Pulmonary tuberculosis: up-to-date imaging and management. Am J Roentgenol. 2008;191:834-44

8) Goo JM, Im JG, Do KH, Yeo JS, Seo JB, Kim HY, Chung JK. Pulmonary tuberculoma evaluated by means of FDG PET: findings in 10 cases. Radiology. 2000;216:117-21

9) Chang JM, Lee HJ, Goo JM, Lee HY, Lee JJ, Chung JK, Im JG False positive and false negative FDG-PET scans in various thoracic diseases Korean J Radiol. 2006;7:57-69

10) Tian G, Xiao Y, Chen B, Guan H, Deng QY. Multi-site abdominal tuberculosis mimics malignancy on 18F-FDG PET/CT: report of three cases World J Gastroenterol. 2010;16:4237-42

11) Smith KR Inaugural article: national burden of disease in India from indoor air pollution. Proc Natl Acad Sci U S A. 2000;97:13286-93

12) Scharko AM, Perlman SB, Pyzalski RW, Graziano FM, Sosman J, Pauza CD Wholebody positron emission tomography in patients with HIV-1 infection Lancet. 2003;362:959-61

13) Sathekge M, Goethals I, Maes A, van de Wiele C Positron emission tomography in patients suffering from HIV-1 infection Eur J Nucl Med Mol Imaging. 2009;36:1176-84

14) Orem J, Mbidde EK, Lambert B, de Sanjose S, Weiderpass E Burkitt's lymphoma in Africa, a review of the epidemiology and etiology. Afr Health Sci. 2007;7:166-75

15) Chen CJ, Lee BF, Yao WJ, Cheng L, Wu PS, Chu CL, Chiu NT Dual-phase 18F-FDG PET in the diagnosis of pulmonary nodules with an initial standard uptake value less than 2.5. Am J Roentgenol. 2008;191:475-9

16) Sathekge MM, Maes A, Pottel H, Stoltz A, van de Wiele C Dual time-point FDG PET$\mathrm{CT}$ for differentiating benign from malignant solitary pulmonary nodules in a TB endemic area. S Afr Med J. 2010;100:598-601

17) Davis SL, Nuermberger EL, Um PK, Vidal C, Jedynak B, Pomper MG, Bishai WR, Jain SKNoninvasive pulmonary [18F]-2-fluoro-deoxy-D-glucose positron emission tomography correlates with bactericidal activity of tuberculosis drug treatment. Antimicrob Agents Chemother. 2009;53:4879-84.

18) Tian G, Xiao Y, Chen B, Xia J, Guan H, Deng Q.FDG PET/CT for therapeutic response monitoring in multi-site non-respiratory tuberculosis Acta Radiol. 2010;51:1002-6

19) .Buscombe JR, Oyen WJ, Corstens FH, Ell PJ, Miller RF Localization of infection in HIV antibody positive patients with fever. Comparison of the efficacy of Ga-67 citrate and radiolabeled human IgG Clin Nucl Med. 1995;20:334-9

20) Buscombe JR, Buttery P, Ell PJ, Miller RF. Patterns of Ga-67 citrate accumulation in human immunodeficiency virus positive patients with and without Mycobacterium avium intracellulare infection Clin Radiol. 1995;50:483-8

21) Schuster DP, Markham J, Welch MJ. Positron emission tomography measurements of pulmonary vascular permeability with Ga-68 transferrin or C-11 methylalbumin Crit Care Med. 1998 Mar;26(3):518-25

22) Prvulovich EM, Miller RF, Costa DC, Severn A, Corbett E, Bomanji J, Becker WS, Ell PJ. Immunoscintigraphy with a 99Tcm-labelled anti-granulocyte monoclonal antibody in patients with human immunodeficiency virus infection and AIDS. Nucl Med Commun. $1995 ; 16: 838-45$

23) Yamada N, Takashima H, Nohara F, Fujinawa T, Matsumura T, Koizumi K, Hisada K. Investigation of $99 \mathrm{mTc}$ labeled tumor seeking agents-usefulness of $99 \mathrm{mTc}$-isoniazid Radioisotopes. 1987;36:505-11

24) Vergne I, Singh S, Roberts E, Kyei G, Master S, Harris J, de Haro S, Naylor J, Davis A, Delgado M, Deretic V Autophagy in immune defense against Mycobacterium tuberculosis. Autophagy. 2006;2:175-8. 